\title{
The effect of gesture on persuasive speech
}

\author{
Judith Peters, Marieke Hoetjes \\ Centre for Language Studies, Radboud University Nijmegen, the Netherlands \\ j.l.petersestudent.ru.nl, m.hoetjes@let.ru.nl
}

\begin{abstract}
Speech perception is multimodal, with not only speech, but also gesture presumably playing a role in how a message is perceived. However, there have not been many studies on the effect that hand gestures may have on speech perception in general, and on persuasive speech in particular. Moreover, we do not yet know whether an effect of gestures may be larger when addressees are not involved in the topic of the discourse, and are therefore more focused on peripheral cues, rather than the content of the message. In the current study participants were shown a speech with or without gestures. Some participants were involved in the topic of the speech, others were not. We studied five measures of persuasiveness. Results showed that for all but one measure, viewing the video with accompanying gestures made the speech more persuasive. In addition, there were several interactions, showing that the performance of the speaker and the factual accuracy of the speech scored high especially for those participants who not only saw gestures but were also not involved in the topic of the speech.
\end{abstract}

Index Terms: multimodal speech perception, gesture, persuasion, dual processing models

\section{Introduction}

Human communication is multimodal. When people speak, they generally use not only speech, but also gestures. In the past few decades it has been established that speech and gesture are closely related $[1,2]$. Despite the ubiquity of gestures in human communication, they are relatively understudied, and although the field of gesture studies has grown, with several hypotheses about the relationship between speech and gesture being proposed [e.g. 3, 4-7], many aspects of the relationship between speech and gesture are still unknown. One of the questions often asked in gesture research is why people gesture when they speak. Studies have suggested that people gesture not only for themselves - to help speech production [8]-, but also for the addressee -to help speech perception [9]. However, most studies have focused on gesture production, and gesture perception has received relatively little attention. The current study focuses on the effect of gesture in speech perception, by studying the effect of gesture production on the perceived persuasiveness of a political speech.

Gestures can be defined as symbolic movements of the hands and arms "related to ongoing talk and to the expressive effort or intention" [10]. This definition excludes self-adaptors, such as touching one's hair or removing an imaginary speck of dust from one's clothes. This definition also indicates that speech and gesture are related. Studies have shown that speech and gesture are closely related at a semantic, pragmatic, and temporal level. For example, it has been shown that gesture is temporally synchronized with co-expressive speech [1]. Also, speech and gesture are acquired together [see 11, for an overview], and break down together in cases of disfluency, for example during stuttering [12]. Additional evidence of the close relationship between speech and gesture has been given by the fact that also congenitally blind people, who have never seen anyone gesture, gesture when they speak [8]. In short, these studies suggest that we cannot get a complete picture of human communication if gesture is not taken into account.

In the present study, we focus on the role of gesture in one specific type of persuasive communication, political speeches. Political speeches differ from more usual face-to-face communication in several ways. Speeches tend to be monologues, and the addressee does not have an active role in the communicative process. Also, one of the main goals of a political speech is to convince addressees about a particular point of view. To this end, speakers can use various pragmatic devices. One possible pragmatic device is using hand gestures while speaking. A question is whether hand gestures play a role in the extent to which such a speech is considered persuasive.

Only a few previous studies have been conducted on the use of gesture in political speeches. Streeck [13] conducted a case study in which he discussed the type of hand gestures that 2004 Democratic Party presidential candidates produced during the primary campaign. He showed that the politicians used many pragmatic gestures, which presumably help the addressee visually structure and process speech. However, since this was a case study, we do not know whether the gestures that the politicians produced actually had an effect on the audience. A study by Maricchiolo et al. [14] did take the audience into account. In their experimental study, they showed participants video clips of a political speech which was either accompanied by one of four types of gestures, or by no gestures. The results were that the participants who saw a video clip with a gesture found the speech more persuasive than if they did not see a gesture. However, the definition of gestures as used in the study by Marrichiolo et al. can be considered problematic (selfadaptors were considered to be gestures). Also, the participants in the study were all involved in the topic of the speech. This could be of importance, because whether an addressee is involved or not might play a role in how a message is processed.

In communication research, several influential message processing models have been proposed. The Elaboration Likelihood Model (ELM) [15] and the Heuristic Systematic Model (HSM) [16] both propose that a recipient can be persuaded by a message in one of two ways, depending on the level of involvement (also called elaboration). If the addressee is highly involved, he or she will consider the quality of the proposed arguments carefully to determine the level of persuasiveness -this is called the central route. If, however, the addressee is not that involved, or even uninterested, the information in the message will be processed more superficially, and not the quality of the proposed arguments, but heuristics affect whether the addressee is persuaded by the 
message - this is called the peripheral route. It might be the case that nonverbal signals such as gestures are one of the heuristics used by addressees who follow the peripheral route.

As far as we are aware, only one previous study has addressed the role of gestures in persuasive communication while also considering the dual processing models. Jackob, Roessing, and Petersen [17] presented students with one of three videos: without vocal emphasis and without gestures, with vocal emphasis but without gestures, or with vocal emphasis and gestures. The speech in the video was evaluated with regard to three dimensions of persuasiveness: performance of the speaker, characteristics of the speaker, and characteristics of the argumentation. It was found that although vocal emphasis and gestures helped increase the perception of some features of the speech, the content of the speech was most important. However, the authors mention that these results can be explained by the fact that it was likely that the participants, who were highly educated and involved students, took the central route. This means that we still do not know what the effect is of gestures in persuasive speeches for addressees who take the peripheral speech processing route.

In the present study the goal is to investigate the effect that hand gestures have in persuasive speeches. We conducted a study in which participants (students) were presented with a speech about a local educational issue. Participants either saw a video of the speech, including the many spontaneous hand gestures that were produced by the speaker, or listened to the speech while viewing several photo stills of the speaker. In addition, some participants were encouraged to take the central processing route, while others were encouraged to take the peripheral processing route. The hypothesis is that viewing hand gestures will make the speech more persuasive for the addressees, in particular when the addressees are not highly involved (i.e. when they take the peripheral route).

\section{Method}

\subsection{Participants}

In total, 116 participants took part in the experiment (112 females, age $M=25, S D=8.35$, range $18-56$ years old). All participants were (part-time) students of pedagogy at a local university of applied sciences, and were unfamiliar with the goal of this study. Participants took part in groups and were randomly allocated to one of the 4 experimental conditions.

\subsection{Material}

Participants were presented with a video of a pre-recorded speech of about 2 minutes. In this speech, the speaker discussed the merits of introducing a new final exam at the local university of applied sciences. The speaker in the video was introduced to the participants as being a local politician (which was not actually the case).

There were 2 versions of the speech (manipulating gesture production). One version (Gesture) used the original sound and video. In the original video the speaker spontaneously produced many hand gestures. The other version of the speech (No Gesture) had the original sound, but instead of the original video, twelve screenshots taken from the video were presented (see figure 1 for an example), one after another, in the course of

\footnotetext{
${ }^{1}$ Note that in this study, a higher score indicated less agreement with the items.
}

the 2-minute speech. These screenshots showed the speaker at moments during the speech when he did not produce any gestures. This means that in the second version, although there was comparable visual input insofar that the same speaker was shown, participants did not see any movement.

The speech was introduced to the participants in one of two ways (manipulating level of elaboration): by either stating that the speech was about an exam which might be introduced at the local university of applied sciences next year, i.e. the participants themselves would have to take this exam (Central condition), or by stating that the exam might be introduced in 2025 , i.e. the participants themselves would not have to take this exam (Peripheral condition).

To check whether participants who thought they might have to take the exam next year actually followed the central processing route, participants, after listening to the speech, were presented with three 7-point Likert scale items on the level of elaboration about the exam $(\alpha=.78$. Example item: The final general exam will influence my life). Participants in the Central condition showed more elaboration $(M=4.5, S D=1.19)$ than participants in the Peripheral condition $(M=2.73, S D=0.97)$, $t_{(112)}=8.85, p<.001$.

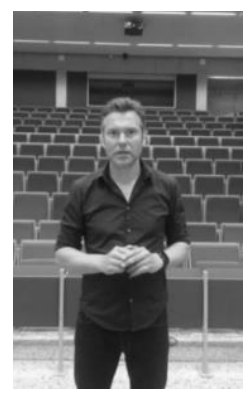

Figure 1: Example screenshot from video material.

\subsection{Instruments}

To analyse persuasion, participants were presented with a questionnaire after they had listened to and viewed the speech. Persuasion was analysed using three dimensions [based on 17]. Each dimension was measured using three 7-point Likert scale items, ranging from 1 (agree completely) to 7 (disagree completely) ${ }^{1}$. We measured

$1)$ the perceived performance of the speaker $(\alpha=.83$. Example item: I thought the speech was lively),

$2)$ the perceived characteristics of the speaker $(\alpha=.85$. Example item: I thought the speaker was competent), and

$3)$ the perceived characteristics of the argumentation $(\alpha=.61$.) Due to this low reliability, the three items of this final dimension were analysed separately (I thought the speech was sincere/factually accurate/interesting).

\subsection{Procedure}

The experiment was conducted in groups. Participants were asked to first read a text introducing the experiment, which contained information about the topic of the speech (the new final exam), including information on when this new exam was to be introduced (depending on the condition, either next year, or 2025). Each group of participants was then presented with 
the audio and, depending on the condition, the video or the screenshots of the speech. After the speech had been played, participants had to fill out the questionnaire individually. Participants were able to ask questions beforehand and were debriefed once the questionnaire had been filled out. The total duration of the experiment for each participant was about 10 minutes.

\subsection{Design and analysis}

The study consisted of a between subjects design, with 2 independent variables: Gesture (levels: Gesture/No gesture) and Elaboration (levels: Central/Peripheral). Persuasion was analysed on three dimensions (see Instruments). Statistical analyses consisted of $2 \times 2$ ANOVAs.

\section{Results}

\subsection{Perceived performance of the speaker}

There was no main effect of Elaboration on the perceived performance of the speaker. However, there was a main effect of Gesture: participants who saw the speech without gestures $(M=4.71, S D=1.22)$ were more negative about the performance of the speaker than the participants who saw the speech with gestures $(M=4.13, S D=1.18), F_{(1,111)}=7.681, p=.007, \eta^{2}=.065$. This main effect was qualified by an interaction between Elaboration and Gesture, $F_{(1,111)}=8.02, p=.006, \eta^{2}=.067$. Participants in the Gesture $\mathrm{x}$ Central condition $(M=4.59$, $S D=1.12$ ) were more negative about the performance of the speaker than participants in the Gesture $\mathrm{x}$ Peripheral condition $(M=3.68, S D=1.07)$, see also figure 2 .

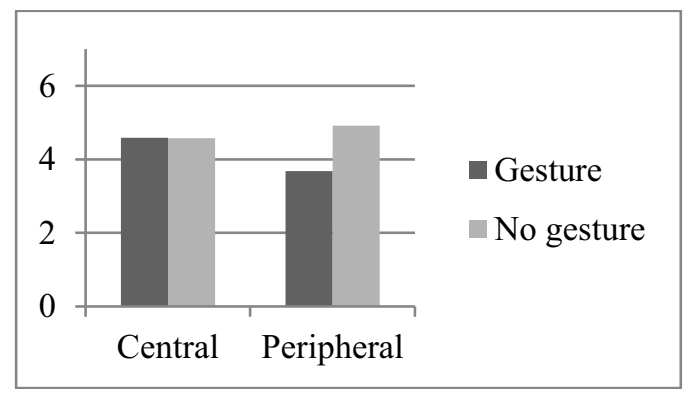

Figure 2: Mean scores for perceived performance of the speaker, for all 4 conditions. A higher score indicates less agreement with the items.

\subsection{Perceived characteristics of the speaker}

There was a main effect of Elaboration on the perceived characteristics of the speaker. Participants in the Central condition $(M=3.60, S D=.93)$ were more negative about the characteristics of the speaker than the participants in the Peripheral condition $(M=2.96, \quad S D=1.02), \quad F_{(1,111)}=11.22$, $p=.001, \eta^{2}=.092$. There was also a main effect of Gesture. Participants who saw the speech without gestures $(M=3.51$, $S D=.94)$ were more negative about the characteristics of the speaker than the participants who saw the speech with gestures $(M=3.07, S D=1.07), F_{(1,111)}=4.609, p=.034, \eta^{2}=.04$. There was no interaction effect between Elaboration and Gesture.

\subsection{Perceived characteristics of the argumentation}

Due to the low reliability $(\alpha=.61$.), the three questions measuring the perceived characteristics of the argumentation were analysed separately.

\subsubsection{Sincerity of the speech}

There was a main effect of Elaboration on the perceived sincerity of the speech. Participants in the Central condition $(M=3.7, S D=1.10)$ found the speech less sincere than participants in the Peripheral condition $(M=3.2, S D=1.20)$, $F_{(1,111)}=5.019, p=.027, \eta^{2}=.043$. There was also a main effect of Gesture. Participants who saw the speech without gestures $(M=3.73, S D=1.10)$ found the speech more insincere than participants who saw the speech with gestures $(M=3.17$, $S D=1.17), F_{(1,111)}=6.81, p=.010, \eta^{2}=.058$. There was no interaction effect between Elaboration and Gesture.

\subsubsection{Factual accuracy of the speech}

There were no main effects of Elaboration or Gesture on the perceived factual accuracy of the speech. There was, however, an interaction effect between Elaboration and Gesture, $F_{(1,11)}=4.72, p=.032, \eta^{2}=041$. Participants in the Gesture $\mathrm{x}$ Central condition $(M=4.12, S D=1.11)$ perceived the speech to be less factually accurate than participants in the Gesture $\mathrm{x}$ Peripheral condition $(M=3.37, S D=1.16)$, see also figure 3 .

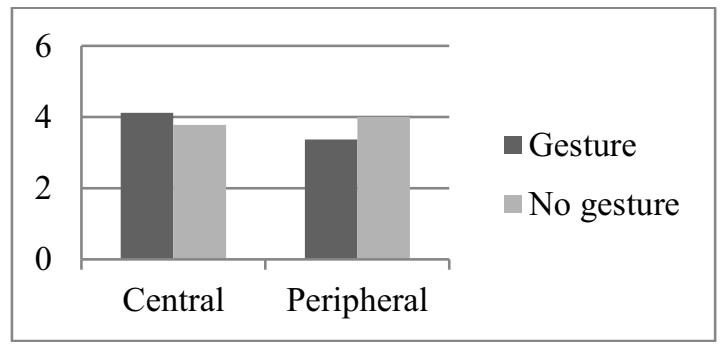

Figure 3: Mean scores for factual accuracy of the speech, for all 4 conditions. A higher score indicates less agreement with the items.

\subsubsection{Interestingness of the speech}

There was no main effect of Elaboration on the perceived interestingness of the speech. There was, however, a main effect of Gesture. Participants who saw the speech without gestures $(M=4.40, S D=1.49)$ found the speech less interesting than participants who saw the speech with gestures $(M=3.70$, $S D=1.45), F_{(1,111)}=6.52, p=.012, \eta^{2}=.055$. There was no interaction effect between Elaboration and Gesture.

\section{Discussion}

This study focused on one aspect of multimodal speech perception, namely hand gestures. The goal of the present study was to study the effect of hand gestures in political speeches on the persuasiveness of the speech. The hypothesis was that viewing hand gestures would make the speech more persuasive for addressees, in particular when the addressees are not highly involved (i.e. when they take the peripheral processing route). The results showed that for four of the five measures, participants who viewed the video without hand gestures indeed found the speech less persuasive than the participants who saw hand gestures while listening to the speech. When there was an 
interaction between level of elaboration and gesture (this was the case for two measures, performance of the speaker and factual accuracy) this interaction was as expected, with a positive effect of gesture only for participants who took the peripheral route.

The results from this study are in line with previous experimental work on the effect of gestures on the perception of persuasive speech [e.g. 14, 17], but extent previous knowledge in an important way. In the study by Maricchiolo et al. [14], and the study by Jackob et al. [17], it was found that viewing gestures made a speech more persuasive. However, in both studies all participants were presumably involved in the topic of the speech. Because gestures can be used as a heuristic cue in message processing, we need to be able to distinguish between people who are carefully considering the content and quality of an argument (and thus take the central processing route), and people who are less involved in a topic and can be persuaded of the quality of a message by heuristic cues (and thus take the peripheral processing route). The present study has made this distinction, and found that for several aspects of persuasiveness, there is only an effect of viewing gestures for people who take the peripheral route. This means that using hand gestures in a political speech can be a useful strategy when trying to persuade people who are not that interested in the topic or who are not paying close attention.

An important alternative explanation of the results is that it may not be the gestures per se, but other aspects of the moving image of the video which made the speech in the gesture condition more persuasive. In the gesture condition, participants were presented with a video, which included gestures, but also all other movements that the speaker made. For example facial expressions and overall body movements could (also) be a reason of someone's increased level of persuasiveness. The setup used in this study cannot exclude this possibility and cannot distinguish between effects of the various nonverbal elements of the speech. Also, we cannot rule out that participants simply considered the gesture condition more fun to watch merely because of watching a moving image, which was in turn reflected in the persuasion measures.

In the current study, it was decided to create the no gesture condition by using the sound of the original video, accompanied by twelve video screenshots. This way, the speech was completely identical in both conditions. As many other aspects as possible were also kept identical: the screenshots in the no gesture condition showed the same speaker in the same context as in the gesture condition. This means that visual aspects such as the appearance of the speaker were kept constant. Participants in the no gesture condition were presented with twelve screenshots in the course of the two minutes that the speech took, so there was also some 'movement' in the no gesture condition. We decided to manipulate the material this way because we wanted the speech to be as natural as possible, and identical between conditions. Creating a no gesture condition by having the speaker produce the same speech without gestures would probably have led to changes in aspects of speech, and could have made the speech less fluent. However, although the current setup had the advantage of having identical speech in both conditions, it had the disadvantage, as mentioned above, of more visual differences between the conditions than only the use of gestures.

To address these points, an alternative setup in future research would be one where the no gesture condition only shows the speaker's face, and not the hands, as compared to the gesture condition where the entire upper body can be shown. Another option would be to use a virtual agent which can be programmed to use gestures, or not. Virtual agents are also one of the domains in which the findings from this study can be applied to speech technology systems.

The current study focused on political speeches. Naturally, there are many other situations in which persuasive speech is used, for example in face to face dialogue. In order to claim that the current findings apply to persuasive speech in general more studies in other persuasive speech domains would have to be done, with similar findings.

Finally, it can be noted that the politician in the video clip was male, and practically all participants in the study were female. It might be the case that male speakers are considered more (or less) persuasive, and it might also be that the gender of the participant plays a role. To determine whether this is the case, future studies could include gender of the speaker and of the participant in the experimental design.

\section{Conclusion}

The goal of this study was to determine whether viewing hand gestures makes a speech more persuasive, in particular when the addressee is not very involved in the topic. Findings firstly showed that a political speech without hand gestures was considered less persuasive than a political speech with hand gestures. Secondly, the results showed that when people were not very involved with the topic, viewing a speech with hand gestures led to more agreement with items measuring perceived performance of the speaker and perceived accuracy of the speech than when perceiving a speech without hand gestures. We can therefore conclude that viewing gestures can positively influence speech perception, and that gestures can be used as a pragmatic device, especially when trying to persuade addressees who are not very involved or not that interested in the topic under discussion.

\section{Acknowledgements}

We would like to thank Raymond Reesink for his help in producing the material, and Peter Berkers and the teachers at the HAN for their help in facilitating this study during teaching hours.

\section{References}

[1] D. McNeill, Hand and mind. What gestures reveal about thought. Chicago: University of Chicago Press, 1992.

[2] A. Kendon, Gesture. Visible action as utterance. Cambridge: Cambridge University Press, 2004.

[3] S. Kita, M. Alibali, and M. Chu, "How do gestures influence thinking and speaking? The Gesture-for-Conceptualization Hypothesis", Psychological Review, vol. 124, pp 245-266, 2017.

[4] S. Kita and A. Özyürek, "What does cross-linguistic variation in semantic coordination of speech and gesture reveal?: Evidence for an interface representation of spatial thinking and speaking", Journal of Memory and Language, vol. 48, pp. 16-32, 2003

[5] R. M. Krauss, Y. Chen, and R.F. Gottesman, "Lexical gestures and lexical access: A process model, in Language and gesture", in D. McNeill (ed.), Language and Gesture, Cambridge University Press: New York, 2000, pp. 261-283.

[6] J. P. de Ruiter, "The production of gesture and speech, in Language and gesture", in D. McNeill (ed.), Language and Gesture, Cambridge University Press: Cambridge, 2000, pp. 284311 . 
[7] A. B. Hostetter and M. Alibali, "Language, gesture, action! A test of the gesture as simulated action framework", Journal of Memory and Language, vol. 63, pp. 245-257, 2010.

[8] J. M. Iverson and S. Goldin-Meadow, "Why people gesture when they speak", Nature, vol. 396, p. 228, 1998.

[9] R. M. Krauss, "Why do we gesture when we speak?", Current Directions in Psychological Science, vol. 7, pp. 54-60, 1998.

[10] M. Gullberg, "Some reasons for studying gesture and second language acquisition (hommage a Adam Kendon)", International Review of Applied Linguistics, vol. 44, pp. 103-124, 2006.

[11] M. Gullberg, K. De Bot, and V. Volterra, "Gestures and some key issues in the study of language development", Gesture, vol. 8, no. 2, pp. 149-179, 2008.

[12] R. I. Mayberry and J. Jaques, "Gesture production during stuttered speech: Insights into the nature of gesture-speech integration", in D. McNeill (ed.), Language and Gesture, Cambridge University Press: New York, 2000, pp. 199-213.

[13] J. Streeck, "Gesture in political communication: A case study of the democratic presidential candidates during the 2004 primary campaign", Research on Language and Social Interaction, vol. 41, no. 2, pp. 154-186, 2008

[14] F. Maricchiolo, F., A. Gnisci, M. Bonaiuto, and G. Ficca, "Effects of different types of hand gestures in persuasive speech on receivers' evaluations", Language and cognitive processes, vol. 24, no. 2, pp. 239-266, 2009.

[15] R. E. Petty and J.T. Cacioppo, Communication and Persuasion Central and Peripheral Routes to Attitude Change. New York: Springer, 1986.

[16] S. Chaiken, "Heuristic versus systematic processing and the use of source versus message cues in persuasion", Journal of Personality and Social Psychology, vol. 39, no. 5, pp. 752-766, 1980.

[17] N. Jackob, T. Roessing, and T. Petersen, "The effects of verbal and nonverbal elements in persuasive communication: Findings from two multi-method experiments", Communications, vol. 36 , pp. $245-271,2011$. 